Tohoku J. Exp. Med., 2007, 211, 133-139

\title{
Cardiac Vagal Activation by Adrenocorticotropic Hormone Treatment in Infants with West Syndrome
}

\author{
Ayako Hattori, ${ }^{1}$ Junichiro Hayano, ${ }^{2}$ Shinji Fujimoto, ${ }^{1}$ Naoki Ando, ${ }^{1}$ \\ Kumiko Mizuno, ${ }^{3}$ Michi Kamei, ${ }^{1}$ Satoru Kobayashi, ${ }^{1}$ Tatsuya Ishikawa ${ }^{1}$ and \\ HAJIME TOGARI ${ }^{1}$ \\ ${ }^{1}$ Department of Pediatrics, Neonatology and Congenital Disorders, Nagoya City University \\ Graduate School of Medical Sciences, Nagoya, Japan \\ ${ }^{2}$ Medical Education, Nagoya City University, Graduate School of Medical Sciences, Nagoya, Japan \\ ${ }^{3}$ Department of Central Clinical Laboratory, Nagoya City University Hospital, Nagoya, Japan
}

Hattori, A., Hayano, J., Fujimoto, S., Ando, N., Mizuno, K., Kamei, M., Kobayashi, S., Ishikawa, T. and Togari, H. Cardiac Vagal Activation by Adrenocorticotropic Hormone Treatment in Infants with West Syndrome. Tohoku J. Exp. Med., 2007, 211 (2), 133-139 - West syndrome (WS) is a generalized epileptic syndrome of infancy and early childhood with various etiologies, and consists of a triad of infantile spasm, arrest or regress of psychomotor development and specific electroencephalogram (EEG) pattern of hypsarrhythmia. WS had been believed to be refractory, but recent evidence supports effectiveness of adrenocorticotropic hormone (ACTH) treatment. The ACTH treatment, however, has a problem that it is often accompanied by adverse autonomic symptoms. We therefore examined heart rate variability (HRV) for assessing cardiac autonomic functions in WS and prospectively observed the changes during ACTH treatment. We studied 15 patients with WS and 9 age-matched controls during sleep (EEG stage 2). Compared with controls, the patients with WS were greater in the low-frequency component (LF) of HRV, an index reflecting sympatho-vagal interaction $(p=0.02)$, but were comparable for high-frequency component (HF) and LF-to-HF ratio (LF/HF), indices reflecting cardiac vagal activity and sympathetic predominance, respectively. During ACTH treatment, heart rate decreased $(p<0.01)$, LF and HF increased $(p<0.01)$, and LF/HF did not differ significantly. These results indicate that West syndrome might be accompanied by autonomic changes and that ACTH treatment enhances parasympathetic function and causes bradycardia. West syndrome; ACTH; heart rate variability; bradycardia; autonomic function (C) 2007 Tohoku University Medical Press

West syndrome (WS) is an age-dependent generalized epileptic syndrome characterized by tonic spasms in clusters and hypsarrhythmia on electroencephalograms (EEG). Adrenocorticotropic hormone $(\mathrm{ACTH})$ is a popular treatment and most effective for WS, but it often induces side effects including autonomic symptoms such as hypertension, irritability and a change in appetite (Dulac and Tuxhorn 2005). Sudden death related to ACTH treatment has also been reported,

Received September 25, 2006; revision accepted for publication December 22, 2006.

Correspondence: Ayako Hattori, M.D., Department of Pediatrics, Neonatology and Congenital Disorders, Nagoya City University Graduate School of Medical Sciences, 1 Kawasumi, Mizuho-cho, Mizuho-ku, Nagoya 467-8601, Japan.

e-mail: aykhat@med.nagoya-cu.ac.jp 
but the mechanism is unclear (Hamano et al. 2005). Although these findings suggest that autonomic abnormalities are involved in the pathophysiology of WS, convincing evidence is not yet available.

The present study investigates whether patients with WS have abnormalities in objective measures of autonomic function and, if so, whether ACTH can modify them. We evaluated autonomic functions by performing spectral analysis of heart rate variability (HRV), which is a physiological arrhythmia generated by autonomic modulations of sinus nodal automaticity that noninvasively and quantitatively assesses autonomic functions. The high-frequency component $(\mathrm{HF}$, at around breathing frequency) of HRV is mediated purely by the vagus and reflects respiratory fluctuation of cardiac vagal activity. The lowfrequency component $(\mathrm{LF}, 0.04-0.15 \mathrm{~Hz})$ is mediated by both sympathetic and vagal activities and thus the LF-to-HF ratio ( $\mathrm{LF} / \mathrm{HF})$ is used as an index of sympathetic relative to vagal predominance (Pomeranz et al. 1985; Pagani et al. 1986; Task Force of the European Society of Cardiology the North American Society of Pacing Electrophysiology 1996). Here, we compared these HRV measures during non rapid eye movement (NREM) sleep stage 2 in EEG between controls and patients with WS. We determined whether HRV values are altered in patients with WS and prospectively observed whether synthetic ACTH can modify them.

\section{Methods}

\section{Patients}

We studied 19 consecutive patients with WS who were less than two years of age. They were admitted to Nagoya City University Hospital between Jan 2003 and Dec 2005 and treated with synthetic ACTH because oral antiepileptic drugs were ineffective against tonic spasms. Patients were excluded if they had congenital cardiac anomalies or atrial fibrillation, or they were prescribed with antiarrhythmic agents. Written informed consent was obtained from at least one of each parents.

\section{Controls}

Nine children aged less than 2 years of age ( 8 boys and 1 girl) who were suspected of having epilepsy but had no significant pathological problems underwent EEG. Two of them were diagnosed as having benign infantile epilepsy, one had febrile convulsion, and six had no neurological abnormalities. None of them had either developmental delay or EEG abnormalities. The mean age of the controls was 8.7 months, which did not differ from that of the patient group.

\section{Protocols}

All procedures of this study were conducted according to the guidelines of the Declaration of Helsinki.

\section{ACTH treatment}

Synthetic corticotrophin $(0.015 \mathrm{mg} / \mathrm{kg}$ daily for symptomatic WS and $0.5 \mathrm{mg}[20 \mathrm{IU}] / \mathrm{m}^{2}$ daily for cryptogenic WS) was administered and continued until the spasms and hypsarrhythmic EEG patterns disappeared. The drug was stopped without tapering if administered for less than 15 days. When the duration of ACTH administration was over 15 days, the same daily dose of ACTH was given once every other day three times. The maximal duration of consecutive ACTH administration was 21 days (Hattori et al. 2006).

\section{EEG and ECG measurements}

We simultaneously measured EEGs and ECGs during NREM sleep stage 2 using a digital EEG polygraph (Nihon Kohden, Tokyo) between 10 a.m. and 3 p.m. Most of the infants received triclofos sodium before the recordings were obtained. None of the children suffered seizures within more than $30 \mathrm{~min}$ before recording. Sleep stage 2 was defined as the presence of $12-14 \mathrm{~Hz}$ spindles. We examined ECGs that were measured for 6 to 8 consecutive min during NREM sleep stage 2 . Among the patients with WS, polygraphic measurements were taken before, on the 7th and last days of administration and one week after the end of ACTH administration.

\section{Analysis of HRV}

The digital ECG data were analyzed using a personal computer. All QRS complexes were initially detected using a fast peak detection algorithm. The results of the analysis were reviewed and any errors in R-wave detection were manually edited. Secondly, all QRS complexes were classified into normal beats (sinus rhythm) or not (ectopic or blocked) and then time series of R-R intervals were generated using only consecutive normal-to-normal intervals. ECGs waveforms were complete and any 
errors were manually corrected.

We calculated HR from mean R-R intervals. To assess HRV, the power spectral density of R-R interval variability was computed by fast Fourier transformation. Thereafter, the power of two frequency regions was computed by integrating the power spectral density within the respective frequency bands; i.e., $0.04-0.15 \mathrm{~Hz}$ for the LF component and $0.30-1.30 \mathrm{~Hz}$ (which covered the breathing frequencies of all subjects) for the HF component. The powers of the LF and HF components were transformed into natural logarithmic values to normalize the distribution of the values.

\section{Statistical analysis}

We used the SPSS (version 13.0) software package for all statistical analysis. The Mann-Whitney's U-test was applied to between-group comparisons. Changes in HR and HRV parameters before, during (the seventh and last days) and after ACTH treatment were analyzed using the one-way analysis of variance (ANOVA) with repeated measures and the Bonferroni method was used to adjust for multiple comparisons. All tests were twotailed; a value of $p<0.05$ was considered statistically significant. Data are presented as means \pm S.D. in the text and as means and S.E. of the mean in the figures.

\section{Results}

\section{Study patients}

Among 19 patients with WS, two were excluded from the study due to congenital cardiac anomalies (large ventricular septal defect and double-outlet right ventricle, respectively) and two others were excluded due to the absence of adequate records. Among 15 patients (9 boys and 6 girls), 11 had symptomatic WS and 4 had cryptogenic WS (Table 1). Their mean age was 9.9 months (range, 5.4-23.4 months) and the mean duration of ACTH treatment was 14.3 days (range, 8-21 days). The administrations of antiepileptic drugs in Table 1 were continued in most patients during ACTH treatment. All patients had no seizures and had no hypsarrhythmia in EEGs on the last day of ACTH and after ACTH. None of patients had subdural hemorrhages after ACTH.

Table 1. Profiles of Patients with West syndrome (WS).

\begin{tabular}{clccc}
\hline $\begin{array}{c}\text { Patient } \\
\text { No. }\end{array}$ & \multicolumn{1}{c}{ Etiology } & $\begin{array}{c}\text { Age at start of ACTH } \\
\text { treatment (months) }\end{array}$ & $\begin{array}{c}\text { Duration of } \\
\text { ACTH (days) }\end{array}$ & $\begin{array}{c}\text { Medication } \\
\text { during study }\end{array}$ \\
\hline 1 & Fetal hypoxia & 8.5 & 11 & ZNS, CZP \\
2 & Periventricular leukomalacia & 8.6 & 14 & ZNS, CLB \\
3 & Periventricular leukomalacia & 14.4 & 14 & ZNS, CZP \\
4 & Aicardi syndrome & 6.5 & 17 & VPA, PB, NZP \\
5 & Holocarboxylase synthetase deficiency & 23.4 & 14 & ZNS, CZP \\
6 & Periventricular leukomalacia & 15.0 & 21 & PB, ZNS, VPA \\
7 & Tuberous sclerosis & 7.2 & 21 & ZNS, CZP, VPA \\
8 & Psychomotor delay with unknown cause & 7.3 & 21 & ZNS \\
9 & Tuberous sclerosis & 14.1 & 14 & ZNS, CZP \\
10 & Neonatal hypoglycemia & 8.1 & 10 & ZNS \\
11 & Psychomotor delay with unknown cause & 10.7 & 14 & ZNS, CZP \\
12 & Cryptogenic & 6.2 & 8 & None \\
13 & Cryptogenic & 5.4 & 12 & ZNS \\
14 & Cryptogenic & 8.0 & 11 & CBZ, ZNS \\
15 & Cryptogenic & 5.8 & 21 & ZNS \\
& & $9.9 \pm 4.9$ & $14 \pm 4$ & \\
\hline
\end{tabular}

ZNS, zonisamide; CZP, clonazepam; CLB, clobazam; VPA, valproate sodium; PB, phenobarbital; NZP, nitrazepam; CBZ, carbamazepine. 
Comparison between WS patients and controls

Differences in HR and HRV measurements between patients with WS and controls were compared using the data before ACTH treatment (Fig. 1). The EEGs in all subjects showed sinus rhythm. The HR did not differ significantly between patients and controls (mean \pm S.D.: $113 \pm$ 15 and $120 \pm 10 \mathrm{bpm}$ ). The LF power was greater in patients with WS than in controls $(5.8 \pm 1.4$ and $4.5 \pm 0.9 \mathrm{ln} \mathrm{ms} \mathrm{ms}^{2}, p=0.02$ ), whereas the difference in the HF power did not reach statistical significance $\left(5.7 \pm 2.0\right.$ and $\left.4.5 \pm 1.4 \mathrm{ln} \mathrm{ms}{ }^{2}\right)$. The $\mathrm{LF} / \mathrm{HF}$ ratio was comparable between patients and controls $(1.7 \pm 1.3$ and $1.7 \pm 1.4)$. Although we compared cryptogenic and symptomatic groups, none of HR, LF power, HF power, and LF/HF ratio before ACTH significantly differed.

\section{Change in HR and HRV measures during} ACTH treatment

Fig. 2 shows the power spectra of the R-R interval in a representative patient with WS before and during ACTH treatment. The power of the HF component was obviously increased during the treatment.

Fig. 3 shows changes in HR and HRV values induced by ACTH. During ACTH treatment, HR was decreased and the LF and HF powers were
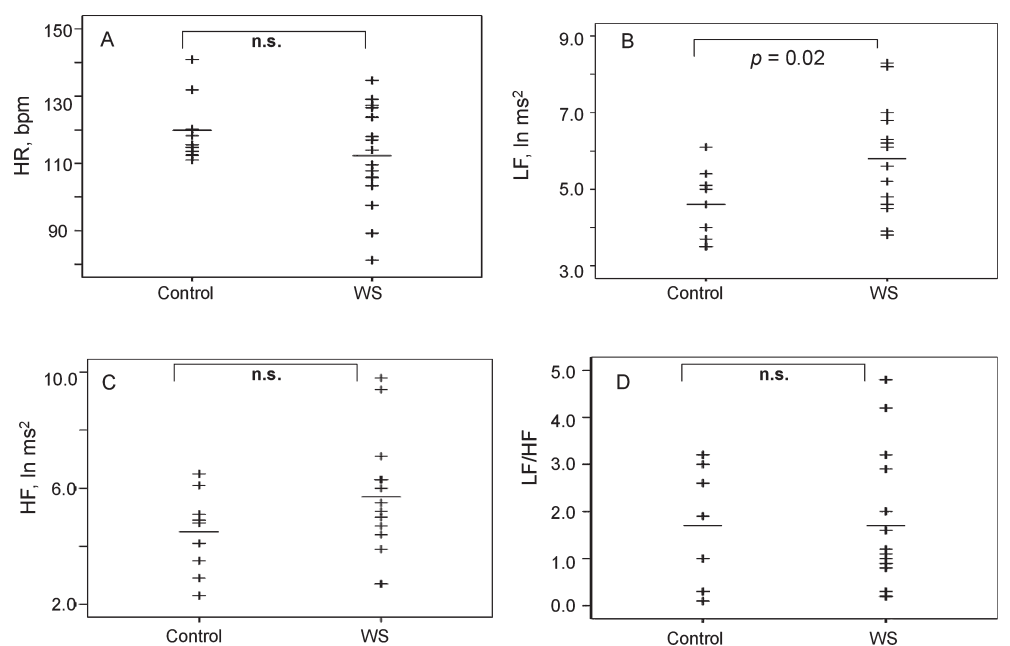

Fig. 1. Comparison of heart rate (HR) and parameters of heart rate variability (HRV) between patients with West syndrome (before ACTH treatment) and controls.
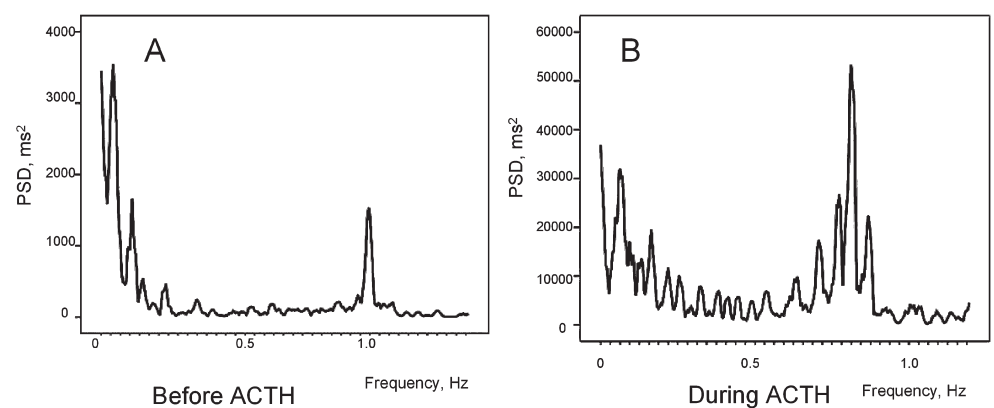

Fig. 2. Power spectral analysis of HRV in a representative patient with West syndrome (A) before and (B) during ACTH treatment. Graphs show power spectra obtained by fast Fourier transformation of $\mathrm{R}$ - $\mathrm{R}$ intervals.

PSD is a power spectral density, valiance per hertz explained by fluctuation at the corresponding frequency. 

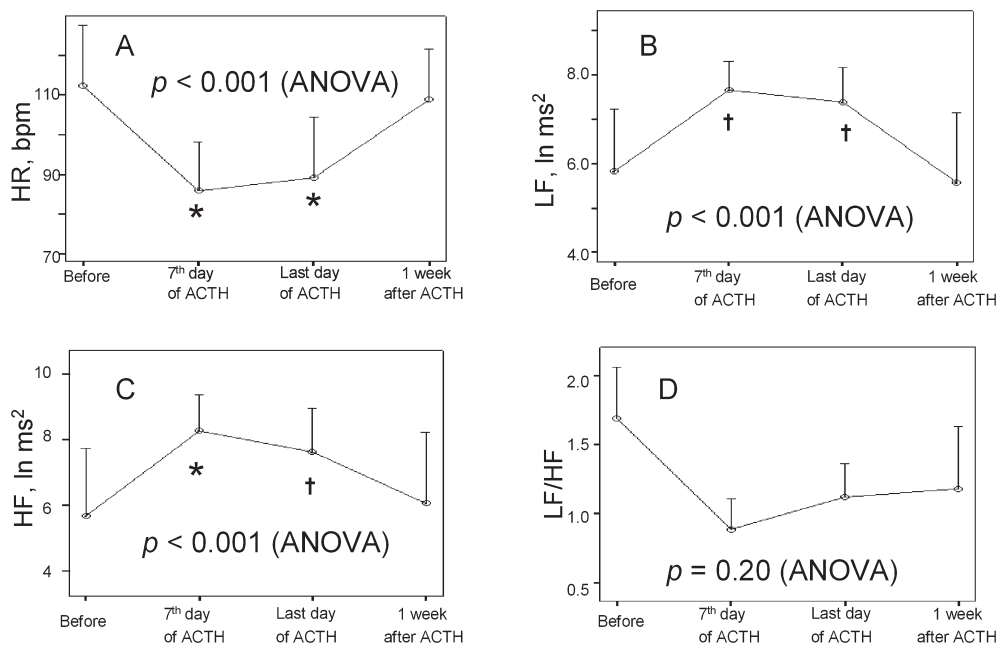

Fig. 3. Graphs show changes in HR and HRV parameters with ACTH treatment. Error bars indicate standard errors (s.e.) of mean. $P$ values indicate significance of the time effect by one-way analysis of variance with repeated measures.

${ }^{*} p<0.001 ; " p<0.01$; significant difference from the value before ACTH treatment (Bonferroni method).

increased $(p<0.001$ for all). These effects were significant at both the seventh and last days of ACTH treatment but were no longer evident one week after the end of treatment. Treatment with ACTH did not significantly change the LH/HF ratio.

\section{Discussion}

We demonstrated here that patients with WS had greater LF power than controls and that ACTH treatment caused a decrease in HR and increases in both LF and HF power of HRV in the patients. The former finding suggests that WS is associated with altered cardiac autonomic function and the latter, that ACTH caused a remarkable increase in cardiac vagal activity and resultant bradycardia in these patients. This is the first study to demonstrate evidence of cardiac vagal activation and bradycardia during ACTH treatment.

We analyzed HRV during sleep stage 2 determined by simultaneous EEG. In general, evaluations of autonomic functions are difficult in infants due to uncooperativeness and/or continuous movement of the subjects. Thus, most previous studies of HRV in this population have been performed using ambulatory ECG or while the subjects are asleep. However, the former could be affected by the level and diurnal pattern of activities and the latter by the stage or quality of sleep (Finley and Nugent 1995; Sanada et al. 1996; Goto et al. 1997; Massin and von Bernuth 1997; Yang et al. 2001; Ferri et al. 2002; Mehta et al. 2002). Given that epilepsy and associated neural disorders affect daily activities and sleep, our method seems advantageous not only for assessing autonomic functions under standardized conditions but also for detecting changes that are directly relevant to diseases or drug effects.

Our observation of increased power of the LF component in patients with WS might reflect a component of autonomic dysfunction that is commonly associated with generalized epilepsies (Devinsky et al. 1994; Opherk et al. 2002; Devinsky 2004). West syndrome is regarded as a form of generalized epilepsy characterized by hypsarrythmia. Evrengul et al. (2005) demonstrated that patients with generalized tonic-clonic seizures have changes in HRV including increased LF power, although they also observed decreased power and increased LF/HF. We believe that etiological factors might not affect autonomic function, because the cryptogenic and symptomatic groups in the present study did not significantly 
differ. However, the distribution of LF power in the WS and control groups overlapped, indicating that some patients with WS do not have autonomic changes. Further study is needed to clarify this point.

The key finding of this study is that ACTH treatment in patients with WS causes bradycardia and changes in HRV that are consistent with cardiac vagal activation. Surprisingly, bradycardia has not been reported as a side effect of ACTH treatment, although Hamano et al. (2005) have described two patients who suddenly died during ACTH treatment. Nevertheless, cardiac vagal activation and resultant bradycardia represent a plausible potential cause of death among patients with epilepsy (Hirsch and Martin 1971; Tennis et al. 1995; Tomson et al. 1998; Nei et al. 2004). Although the administration of antiepileptic drugs except for ACTH was not changed and HR and HRV parameters did not significantly differ between before and after ACTH, the HR and HRV parameters were altered during ACTH treatment. These observations indicate that the pharmacokinetic action of ACTH affects autonomic function.

The mechanisms of vagal activation during ACTH treatment remain undetermined, but the suppression of corticotropin-releasing hormone (CRH) by ACTH might be involved. The systemic administration of ACTH decreases the expression of $\mathrm{CRH}$, which stimulates sympathetic outflow and inhibits parasympathetic outflow (Brown et al. 1982; Fisher et al. 1983; Wiersma et al. 1993; Brunson et al. 2001; Wang et al. 2004). These facts suggest that ACTH enhances vagal activity in patients with WS at least partly through $\mathrm{CRH}$ suppression.

Others have found that 3 to $5 \%$ of patients treated with ACTH die and that these patients tend to have received large doses of ACTH (Riikonen 2001; Hamano et al. 2004). Most protocols of ACTH treatment for WS indicate that ACTH should be administered for more than 14 days and then tapered (Yanagaki et al. 1999; Mackay et al. 2004). Because longer ACTH treatment is associated with more side effects including hypertension, infection, subdural hemorrhage and brain atrophy, some investigators have rec- ommended a shorter duration of ACTH treatment (Ito et al. 1990; Hrachovy et al. 1994; Kondo et al. 2005; Oguni et al. 2006).

In addition to these known side effects, the present study also suggests that obvious cardiac vagal activation and bradycardia are also important side effects that require more clinical consideration. Furthermore, we found that these effects appear within 7 days of ACTH treatment and persist until the end of treatment. However, these effects seem reversible as they disappeared within a week after withdrawing treatment.

In conclusion, we identified changes in cardiac autonomic functions in patients administered with ACTH, by analyzing HRV under the standardized conditions of sleep stage 2. Consistent with other generalized types of epilepsy our findings indicated that WS is associated with altered autonomic functions and that ACTH treatment in patients with WS causes marked cardiac vagal activation and resultant bradycardia. These potential mechanisms could explain some of the adverse effects of ACTH therapy.

\section{References}

Brown, M.R., Fisher, L.A., Spiess, J., Rivier, C., Rivier, J. \& Vale, W. (1982) Corticotropin-releasing factor: actions on the sympathetic nervous system and metabolism. Endocrinology, 111, 928-931.

Brunson, K.L., Khan, N., Eghbal-Ahmadi, M. \& Baram, T.Z. (2001) Corticotropin (ACTH) acts directly on amygdala neurons to down-regulate corticotropin-releasing hormone gene expression. Ann. Neurol., 49, 304-312.

Devinsky, O., Perrine, K. \& Theodore, W.H. (1994) Interictal autonomic nervous system function in patients with epilepsy. Epilepsia, 35, 199-204.

Devinsky, O. (2004) Effects of Seizures on Autonomic and Cardiovascular Function. Epilepsy Curr., 4, 43-46.

Dulac, O. \& Tuxhorn, I. (2005) Infantile spasms and West syndrome. In: Epileptic Syndromes in Infancy, Childhood and Adolescence, 4th ed., edited by J. Roger, M. Bureau, C. Dravet, P. Genton, C.A. Tassinari \& P. Wolf, John Libbey Eurotext, pp. 55-59.

Evrengul, H., Tanriverdi, H., Dursunoglu, D., Kaftan, A., Kuru, O., Unlu, U. \& Kilic, M. (2005) Time and frequency domain analyses of heart rate variability in patients with epilepsy. Epilepsy Res., 63, 131-139.

Ferri, R., Curzi-Dascalova, L., Arzimanoglou, A., Bourgeois, M., Beaud, C., Nunes, M.L., Elia, M., Musumeci, S.A. \& Tripodi, M. (2002) Heart rate variability during sleep in children with partial epilepsy. J. Sleep. Res., 11, 153-160.

Finley, J.P. \& Nugent, S.T. (1995) Heart rate variability in infants, children and young adults. J. Auton. Nerv. Syst., 9, 103-108.

Fisher, L.A., Jessen, G. \& Brown, M.R. (1983) Corticotropin- 
releasing factor (CRF): mechanism to elevate mean arterial pressure and heart rate. Regul. Pept., 5, 153-161.

Goto, M., Nagashima, M., Baba, R., Nagano, Y., Yokota, M., Nishibata, K. \& Tsuji, A. (1997) Analysis of heart rate variability demonstrates effects of development on vagal modulation of heart rate in healthy children. J. Pediatr., 130, 725-729.

Hamano, S., Mochizuki, M., Tanaka, M., Yamashita, S., Minamitani, M., Hishitani, T., Saito, M. \& Eto, Y. (2004) Motrtality and cause of death in patients with West syndrome. Nihon Shonika Gakkai Zasshi, 108, 859-863. (In Japanese with English abstract)

Hamano, S., Tanaka, M., Mochizuki, M., Yamashita, S., Minamitani, M., Hishitani, T., Ogawa, K. \& Akashi, S. (2005) Death associated with corticotropin therapy in West syndrome. Saitama-ken Igakukai Zasshi, 39, 603-606. (In Japanese)

Hattori, A., Ando, N., Hamaguchi, K., Hussein, MH., Fujimoto, S., Ishikawa, T. \& Togari, H. (2006) Short-Duration ACTH therapy for cryptogenic West syndrome with better outcome. Pediatr Neurol., 35, 415-418.

Hirsch, C.S. \& Martin, D.L. (1971) Unexpected death in young epileptics. Neurology, 21, 682-690.

Hrachovy, R.A., Frost, J.D. \& Glaze, D.G., Jr. (1994) Highdose, long-duration versus low-dose, short-duration corticotropin therapy for infantile spasms. J. Pediatr., 124, 803-806.

Ito, M., Okuno, T., Fujii, T., Mutoh, K., Oguro, K., Shiraishi, H., Shirasaka, Y. \& Mikawa, H. (1990) ACTH therapy in infantile spasms: relationship between dose of ACTH and initial effect or long-term prognosis. Pediatr. Neurol., 6, 240-244.

Kondo, Y., Okumura, A., Watanabe, K., Negoro, T., Kato, T., Kubota, T. \& Kakizawa, H. (2005) Comparison of two low dose ACTH therapies for West syndrome: their efficacy and side effect. Brain Dev., 27, 326-330.

Mackay, M.T., Weiss, S.K., Adams-Webber, T., Ashwal, S., Stephens, D., Ballaban-Gill, K., Baram, T.Z., Duchowny, M., Hirtz, D., Pellock, J.M., Shields, W.D., Shinnar, S., Wyllie, E. \& Snead, O.C., III. (2004) Practice parameter: medical treatment of infantile spasms: report of the American Academy of Neurology and the Child Neurology Society. Neurology, 62, 1668-1681.

Massin, M. \& von Bernuth, G. (1997) Normal ranges of heart rate variability during infancy and childhood. Pediatr. Cardiol., 18, 297-302.

Mehta, S.K., Super, D.M., Connuck, D., Salvator, A., Singer, L., Fradley, L.G., Harcar-Sevcik, R.A., Kirchner, H.L. \& Kaufman, E.S. (2002) Heart rate variability in healthy newborn infants. Am. J. Cardiol., 89, 50-53.

Nei, M., Ho, R.T., Abou-Khalil, B.W., Drislane, F.W., Liporace, J., Romeo, A. \& Sperling, M.R. (2004) EEG and ECG in sudden unexplained death in epilepsy. Epilepsia, 45, $338-345$.

Oguni, H., Yanagaki, S., Hayashi, K., Imai, K., Funatsuka, M.,
Kishi, T. \& Osawa, M. (2006) Extremely low-dose ACTH step-up protocol for West syndrome: maximum therapeutic effect with minimal side effects. Brain. Dev., 28, 8-13.

Opherk, C., Coromilas, J. \& Hirsch, L.J. (2002) Heart rate and EKG changes in 102 seizures: analysis of influencing factors. Epilepsy Res., 52, 117-127.

Pagani, M., Lombardi, F., Guzzetti, S., Rimoldi, O., Furlan, R., Pizzinelli, P., Sandrone, G., Malfatto, G., Dell'Orto, S. \& Piccaluga, E. (1986) Power spectral analysis of heart rate and arterial pressure variabilities as a marker of sympathovagal interaction in man and conscious dog. Circ. Res., 59, 178-193.

Pomeranz, B., Macaulay, R.J., Caudill, M.A., Kutz, I., Adam, D., Gordon, D., Kilborn, K.M., Barger, A.C., Shannon, D.C. \& Cohen, R.J. (1985) Assessment of autonomic function in humans by heart rate spectral analysis. Am. J. Physiol., 248, 151-153.

Riikonen, R. (2001) Long-term outcome of patients with West syndrome. Brain Dev., 23, 683-687.

Sanada, M., Yokota, S., Yuza, N., Nishida, T., Itoh, T., Ohishi, T., Ogawa, K., Hamano, S. \& Nara, T. (1996) Assessment of autonomic nerve-function in West syndrome by heart rate variability. Saitama-Shouni Iryou Center Igakushi, 13, 5-8. (In Japanese with English summary)

Task Force of the European Society of Cardiology the North American Society of Pacing Electrophysiology (1996) Heart rate variability-standards of measurement, physiological interpretation, and clinical use. Circulation, 93, 1043-1065.

Tennis, P., Cole, T.B., Annegers, J.F., Leestma, J.E., McNutt, M. \& Rajput, A. (1995) Cohort study of incidence of sudden unexplained death in persons with seizure disorder treated with antiepileptic drugs in Saskatchewan, Canada. Epilepsia, 36, 29-36.

Tomson, T., Ericson, M., Ihrman, C. \& Lindblad, L.E. (1998) Heart rate variability in patients with epilepsy. Epilepsy Res., 30, 77-83.

Wang, W., Murphy, B., Dow, K.E., David, A. R. \& Fraser, D.D. (2004) Systemic adrenocorticotropic hormone administration down-regulates the expression of corticotropin-releasing hormone $(\mathrm{CRH})$ and $\mathrm{CRH}$-binding protein in infant rat hippocampus. Pediatr. Res., 55, 604-610.

Wiersma, A., Bohus, B. \& Koolhaas, J.M. (1993) Corticotropin-releasing hormone microinfusion in the central amygdala diminishes a cardiac parasympathetic outflow under stress-free conditions. Brain Res., 22, 219-227.

Yanagaki, S., Oguni, H., Hayashi, K., Imai, K., Funatuka, M., Tanaka, T., Yanagaki, M. \& Osawa M. (1999) A comparative study of high-dose and low-dose ACTH therapy for West syndrome. Brain Dev., 21, 461-467.

Yang, T., Wong, T., Chang, K., Kwan, S., Kuo, W., Lee, Y. \& Kuo, T.B. (2001) Power spectrum analysis of heart rate variability in children with epilepsy. Childs Nerv. Syst., 17, 602-606. 